\title{
Value of FDG-PET in detecting residual or recurrent nonsmall cell lung cancer
}

\author{
Th. Bury*, J.L. Corhay*, B. Duysinx*, F. Daenen**, B. Ghaye ${ }^{+}$, \\ N. Barthelemy ${ }^{++}$, P. Rigo**, P. Bartsch*
}

Value of FDG-PET in detecting residual or recurrent nonsmall cell lung cancer. Th. Bury, J.L. Corhay, B. Duysinx, F. Daenen, B. Ghaye, N. Barthelemy, P. Rigo, P. Bartsch. (C)ERS Journals Ltd 1999.

ABSTRACT: In order to evaluate the usefulness of 18-fluorodeoxyglucose (FDG) positron emission tomography (PET) in the assessment of therapeutic effects, a study was performed before and after therapy in $\mathbf{1 2 6}$ patients with non-small cell lung cancer (NSCLC) codified stage I to stage IIIB.

Treatment with an early curative result was given in 58 patients, whereas in 68 cases it was limited to palliation. During the treatment follow-up period ( $8-40$ months), each patient was evaluated every 3 months by clinical examination and $\leq 6$ months by imaging techniques (PET and computed tomography (CT)).

A diagnosis of persistent or recurrent tumour was established by means of pathological analysis in 31 patients and by clinical evolution and subsequent imaging progression in 29 other patients. PET showed increased FDG uptake in all cases $(n=60)$ of persistent or recurrent tumour, whereas CT was nonspecific in 17 cases. Conversely, there were five false positive cases via PET imaging and three via CT. In detecting residual or recurrent NSCLC, PET had a sensitivity of $100 \%$ and specificity of $92 \%$, whereas CT had a sensitivity and specificity of $71 \%$ and $95 \%$ respectively.

In conclusion, 18-fluorodeoxyglucose positron emission tomography correctly identified response to therapy in $96 \%$ (121 of 126) of patients. Positron emission tomography appears to be more accurate $(p=0.05)$ than conventional imaging in distinguishing persistent or recurrent tumour from fibrotic scar in patients undergoing treatment for non-small cell lung cancer.

Eur Respir J 1999; 14: 1376-1380.

One major clinical problem in patients treated for lung cancer is the evaluation of treatment effect and the detection of recurrence. It is often difficult to determine post-therapy tumour viability status as the anatomical information provided by chest radiographs and computed tomography cannot clearly distinguish necrotic tumour or fibrotic scar tissue from residual or recurrent tumour. Examination of biopsy material may also be inconclusive because of limited sampling problems. Furthermore, pathological assessment provides information at a single time-point and is not suitable for sequential assessment.

Positron emission tomography (PET) with 18-fluorodeoxyglucose (FDG) makes use of a fundamental biochemical difference in glucose metabolism between normal and neoplastic cells to differentiate benign from malignant tissue [1-3]. In previous clinical reports, the value of FDGPET in the management of patients with unspecified solitary pulmonary nodules and in the preoperative staging of patients with known non-small cell lung cancer (NSCLC) has been shown [4-6].

In this study, the use of FDG-PET for the differentiation of residual or recurrent tumour from post-treatment changes in 126 patients with NSCLC codified stage I to IIIB who had undergone local treatment (surgery and/or radiation therapy) occasionally associated with chemotherapy was evaluated. The results of the FDG-PET studies are reported here and compared with those provided by a conventional morphological imaging technique.

\section{Patients and methods}

One hundred and fifty-two patients with potentially resectable NSCLC were examined at the authors' hospital between September 1994 and April 1997. From this group, 19 patients with poor physiological status and a Karnofsky index $<60$ were promptly excluded from analysis. Wholebody FDG-PET and conventional imaging methods (chest and abdominal computed tomography (CT) and bone scintigraphy) were performed to determine the extent of tumour invasion. Seven patients who died within 6 months and for whom therefore incomplete data were available were also excluded from the final analysis. The final analysis included 126 patients ( 78 males and 48 females; age range 38-81 yrs). A histological diagnosis of the primary tumour was established in all patients: 61 had squamous cell carcinoma, 49 adenocarcinoma (including nine bronchioloalveolar carcinoma), 10 adenosquamous cell carcinoma and six undifferentiated large cell carcinoma. The final staging of the disease was as follows: stage I in 20 patients, stage II 
Table 1. - Therapeutic regimens in 126 patients with nonsmall cell lung cancer

\begin{tabular}{lccc}
\hline Stage & Tumour staging & Patients $\mathrm{n}$ & Treatment \\
\hline Curative group (n=58) & & \\
I & T1-2 N0 & 20 & $\mathrm{~S}$ \\
II & T1-2 N1 (SCC) & 12 & $\mathrm{~S}$ \\
& T1-2 N1 (Adeno) & 8 & $\mathrm{~S}+\mathrm{R}$ \\
IIIA & T3 N0 & 2 & $\mathrm{C}+\mathrm{R}$ \\
& T3 N1 & 4 & $\mathrm{~S}+\mathrm{R}$ \\
Palliative group (n=68) & 12 & $\mathrm{C}+\mathrm{S}$ \\
\multicolumn{1}{c}{ IIIA } & T3 N2* & 9 & $\mathrm{C}+\mathrm{S}$ \\
IIIB & T1-3 N2** & 10 & $\mathrm{R}+\mathrm{C}$ \\
& T4 N0-2 & 9 & $\mathrm{R}+\mathrm{C}$ \\
& & 5 & $\mathrm{R}$ \\
& T1-4 N3 & 26 & $\mathrm{R}$ \\
& & 9 & $\mathrm{R}+\mathrm{C}$ \\
\hline
\end{tabular}

*: limited disease; ${ }^{* *}$ : extensive disease. T: primary tumour; $\mathrm{N}$ : regional nodes; S: surgery; R: radiotherapy; C: chemotherapy; SCC: squamous cell carcinoma; Adeno: adenocarcinoma.

in 22 patients, stage IIIA in 35 patients, and stage IIIB in 49 patients [7].

Patients were classified into two groups (curative or palliative) according to two criteria: the initial staging of the disease, and the early tumour response to treatment.

Treatment with an early apparent curative result was given in 58 cases, whereas in 68 cases it was limited to palliation (table 1). Patients included in the study were followed, after the end of the treatment, for $\geq 8$ months and $\leq 40$ months. Each patient was evaluated every 3 months during the treatment follow-up period. A clinical examination was performed at each visit and the followup imaging (CT and PET) at 6-month intervals, or earlier if a symptom was present. When clinical examination or imaging suggested residual or recurrent tumour, confirmation was obtained by means of biopsy or clinical/ radiological follow-up.

CT was performed using a PQ 2000 (fourth generation; Picker, Cleveland, OH, USA); PET was performed using a PENN PET 240H scanner (UGM, Philadelphia, PA, USA) 60 min post-FDG injection, as previously described [4, 5]. Transmission scans were obtained for attenuation correction in 35 instances. In these 35 instances, FDG uptake was expressed as standardized uptake value (SUV), where the SUV is the tissue concentration in $\mathrm{nCi} \cdot \mathrm{g}^{-1}$ (injected does.body weight ${ }^{-1}$ ). In addition, all PET data were classically analysed by visual interpretation of coronal, sagittal and transverse slices alone and by cross-reference.

PET and CT were interpreted separately by two nuclear physicians or two radiologists and the results then compared to each other and in pertinent cases with histological data. A biopsy of suspected lesions was carried out whenever possible. In other cases, imaging abnormalities were considered positive for tumour when the clinical and radiological follow-up data were strongly suggestive: symptomatic patient and subsequent abnormality progression on chest radiography. In the absence of a suspected lesion, clinical and radiological follow-up were obtained over a period of $\geq 6$ months before considering the case as a true negative result.

The study was approved by the Ethics Committee of the University of Liège.

\section{Statistical analysis}

Sensitivity, specificity, positive and negative predictive value (PPV and NPV, respectively) and accuracy of PET imaging in detecting recurrent lung cancer were determined by correlating PET diagnoses with pathological results in 31 lesions and with clinical outcomes in all other cases. The 95\% confidence interval (CI) was given for the accuracy value. To assess the agreement between CT or $\mathrm{PET}$ and final diagnosis more precisely, the Cohen $\mathrm{K}$ with its $95 \%$ CI was calculated for the same patients [8]. This $\mathrm{K}$ allows comparison of the degree of agreement between the two methods. By means of this statistical method, the degree of agreement between two methods is better if $\mathrm{K}$ approaches 1 . To compare the diagnostic efficacy of CT and PET, their $\mathrm{K}$ were compared using Chi-squared analysis. A p-value $\leq 0.05$ was accepted as significant.

\section{Results}

\section{Treatment with an early apparent curative result}

Treatment with an apparent curative result was given in 42 patients with stage I or II disease and in 16 patients with stage IIIA disease (table 1). None of these patients had a persistent intrathoracic radiographic abnormality 3 months after the end of treatment. All these patients underwent sequential thoracic PET and CT in the post-therapy followup period (mean duration 30 months). To date, 13 recurrent tumours confirmed by biopsy in seven asymptomatic patients and by clinical course with radiographic confirmation in six symptomatic patients (mean time of recurrence 15 months) were observed. A comparison of PET and CT results showed discordant finding in six $(10.3 \%)$ of 58 patients, whereas concordant imaging results were observed in 52 patients. Table 2 summarizes the six discordant CT and PET results in patients treated with curative intent. By comparison to the final diagnosis (histology and radiological follow-up), PET results were correct in five cases and CT results in one. All tumour recurrences $(n=13)$ were correctly diagnosed by PET which showed intense FDG uptake in the lesion (table 3). By contrast, four mediastinal (regional nodes $(\mathrm{N}) 2$ disease) recurrences were not correctly diagnosed by means of CT in four asymptomatic patients. Retreatment with external irradiation or chemotherapy was given to these 13 patients with the objective of obtaining prolonged control of their limited stage of recurrent disease. They were followed up for $>12$ months after completion of retreatment. Twelve of these 13 patients had no relapse but one died of disseminated metastases.

PET and CT correctly identified the absence of tumour recurrence in 44 of 45 patients. There was one false positive case by PET imaging in a patient with radiation pneumonitis but also one false positive case by CT (table 2). At present, PET, for the detection of recurrent tumour in patients who have been treated with early apparent curative result, has a sensitivity of $100 \%$, a specificity of $97 \%$, a PPV of $93 \%$, an NPV of $100 \%$ and a diagnostic accuracy of $98 \%$ (95\% CI $90-99 \%)$. By comparison, the accuracy of CT in this analysis was $91 \%$ (95\% CI $81-$ 97\%). 
Table 2. - Discordant positron emission tomography (PET)/computed tomography (CT) findings following therapy with curative intent in six patients with non-small cell lung cancer

\begin{tabular}{|c|c|c|c|c|c|}
\hline \multirow[b]{2}{*}{$\begin{array}{l}\text { Patient } \\
\text { No }\end{array}$} & \multirow[b]{2}{*}{$\begin{array}{l}\text { Cell } \\
\text { type }\end{array}$} & \multirow[b]{2}{*}{$\begin{array}{c}\text { Local } \\
\text { treatment }\end{array}$} & \multicolumn{3}{|c|}{ Results } \\
\hline & & & PET & CT & Final diagnosis \\
\hline 1 & Adeno & $\mathrm{C}+\mathrm{S}$ & $\begin{array}{l}\text { Increased FDG uptage in mediastinal } \\
\text { node } 12 \text { months post-treatment (TP) }\end{array}$ & Negative (FN) & Recurrence \\
\hline 2 & $\mathrm{SCC}$ & $\mathrm{S}+\mathrm{R}$ & $\begin{array}{l}\text { Increased FDG uptage in area of } \\
\text { fibrosis } 6 \text { months post-treatment (FP) }\end{array}$ & Postradiation pneumonia (TN) & Fibrosis \\
\hline 3 & Adeno & $\mathrm{C}+\mathrm{S}$ & $\begin{array}{l}\text { Increased FDG uptage in mediastinal } \\
\text { node } 6 \text { months post-treatment (TP) }\end{array}$ & Negative (FN) & Recurrence \\
\hline 4 & Adeno & $\mathrm{S}+\mathrm{R}$ & Negative study (TN) & $\begin{array}{c}\text { Tissue changes suspicious for tumour } \\
18 \text { months post-treatment (FP) }\end{array}$ & Fibrosis \\
\hline 5 & Adeno & $\mathrm{S}+\mathrm{R}$ & $\begin{array}{l}\text { Increased FDG uptake in mediastinal } \\
\text { node } 9 \text { months post-treatment (TP) }\end{array}$ & Negative $(\mathrm{FN})$ & Recurrence \\
\hline 6 & $\mathrm{SCC}$ & $\mathrm{S}$ & $\begin{array}{l}\text { Increased FDG uptage in mediastinal } \\
\text { node } 12 \text { months post-treatment (TP) }\end{array}$ & Negative (FN) & Recurrence \\
\hline
\end{tabular}

Adeno: adenocarcinoma; SCC: squamous cell carcinoma; C: chemotherapy; S: surgery; R: radiotherapy; FDG: 18-fluorodeoxyglucose; TP: true positive; FP: false positive; TN: true negative; FN: false negative.

\section{Treatment with an early apparent palliative result}

In this group of patients $(n=68)$, the therapeutic regimens were as follows: chemotherapy plus surgery $(n=9)$; radiotherapy plus chemotherapy $(\mathrm{n}=28)$; and radiotherapy $(n=31)$. All of these patients had a persistent intrathoracic radiographic abnormality 3 months after the end of treatment. A diagnosis of persistent or recurrent carcinoma was established by means of pathological analysis in 24 patients and by documented clinical and radiographic progression of the disease in 23 patients. The condition of the remaining 21 patients remained stable without demonstration of recurrent disease for $\geq 15$ months after treatment. All cases $(n=47)$ of recurrent or persistent lung tumour demonstrated increased FDG uptake, whereas CT results were nonspecific (stable appearance and no evidence of tumour progression) in 13 cases (table 4). Twenty-one patients showed no evidence of recurrent tumour 15-30 months (mean 20 months) after treatment; they were correctly identified by PET in 17 cases and by CT in 19 . Indeed, in this group of patients, there were four false positive cases by PET (two infectious processes and two radiation pneumonitis) and two by $\mathrm{CT}$. The two cases of radiation pneumonitis were apparent on chest radiography 6 months after treatment; in the first case, FDG uptake in the area of fibrosis was moderate (SUV of 3), whereas it was intense (SUV of 7) in the second case.

Table 3. - Imaging results from the follow-up of the 58 patients in the early curative group: correlation with final diagnosis

\begin{tabular}{lrc}
\hline & \multicolumn{2}{c}{ Final diagnosis } \\
\cline { 2 - 3 } & Remission & Recurrent disease \\
\hline FDG-PET & & \\
Negative & $44(\mathrm{TN})$ & $0(\mathrm{FN})$ \\
Positive & $1(\mathrm{FP})$ & $13(\mathrm{TP})$ \\
CT & & $4(\mathrm{FN})$ \\
Negative & $44(\mathrm{TN})$ & $9(\mathrm{TP})$ \\
Positive & $1(\mathrm{FP})$ & \\
\hline
\end{tabular}

FDG-PET: 18-fluorodeoxyglucose positron emission tomography; CT: computed tomography; TN: true negative; FN: false negative; FP: false postive; TP: true positive.
Subsequently, the intensity of FDG uptake in the areas of radiation pneumonitis decreased to a normal level (SUV $<2.5)$ at 12 months in the first case and at 18 months postirradiation in the second case. The false positive cases by CT consisted of tissue changes suspicious for tumour recurrence 12 months postradiotherapy, whereas FDG and anatomical analysis gave negative results.

Figure 1 illustrates the case of a 74-yr-old female with stage III NSCLC treated by chemotherapy and radiotherapy and shows the discordance between CT and PET findings during follow-up.

At present, PET, for the detection of persistent or recurrent tumour in patients who have been treated with palliative intent, has a diagnostic accuracy of $94 \%$ (95\% CI $85-98 \%$ ), a sensitivity of $100 \%$, a specificity of $81 \%$, a NPV of $100 \%$ and a PPV of $92 \%$. By comparison, the accuracy of CT in the present analysis was $78 \%(95 \%$ CI $66-87 \%)$.

Table 5 summarizes the comparative performances of $\mathrm{CT}$ and PET in the detection of residual or recurrent NSCLC in the 126 patients. By comparing the $\mathrm{K}$ of CT $(\mathrm{K}=$ $0.68 ; 95 \% \mathrm{CI} 0.5-0.85)$ and that of PET $(\mathrm{K}=0.92$; $95 \% \mathrm{CI}$ $0.75-1)$, it can be concluded that the agreement between PET and final diagnosis was significantly better than the agreement between CT and final diagnosis $(\mathrm{p}=0.05)$.

In this study, 73 of 126 patients received treatment with radiotherapy. Following radiotherapy, changes on chest

Table 4. - Imaging results from the follow-up of the 68 patients in the early curative group: correlation with final diagnosis

\begin{tabular}{lrc}
\hline & \multicolumn{2}{c}{ Final diagnosis } \\
\cline { 2 - 3 } & Remission & Residual or recurrent disease \\
\hline FDG-PET & & \\
Negative & $17(\mathrm{TN})$ & $0(\mathrm{FN})$ \\
Positive & $4(\mathrm{FP})$ & $47(\mathrm{TP})$ \\
CT & & $13(\mathrm{FN})$ \\
Negative & $19(\mathrm{TN})$ & $34(\mathrm{TP})$ \\
Positive & $2(\mathrm{FP})$ & \\
\hline
\end{tabular}

FDG-PET: 18-fluorodeoxyglucose positron emission tomography; CT: computed tomography; TN: true negative; FN: false negative; FP: false postive; TP: true positive. 

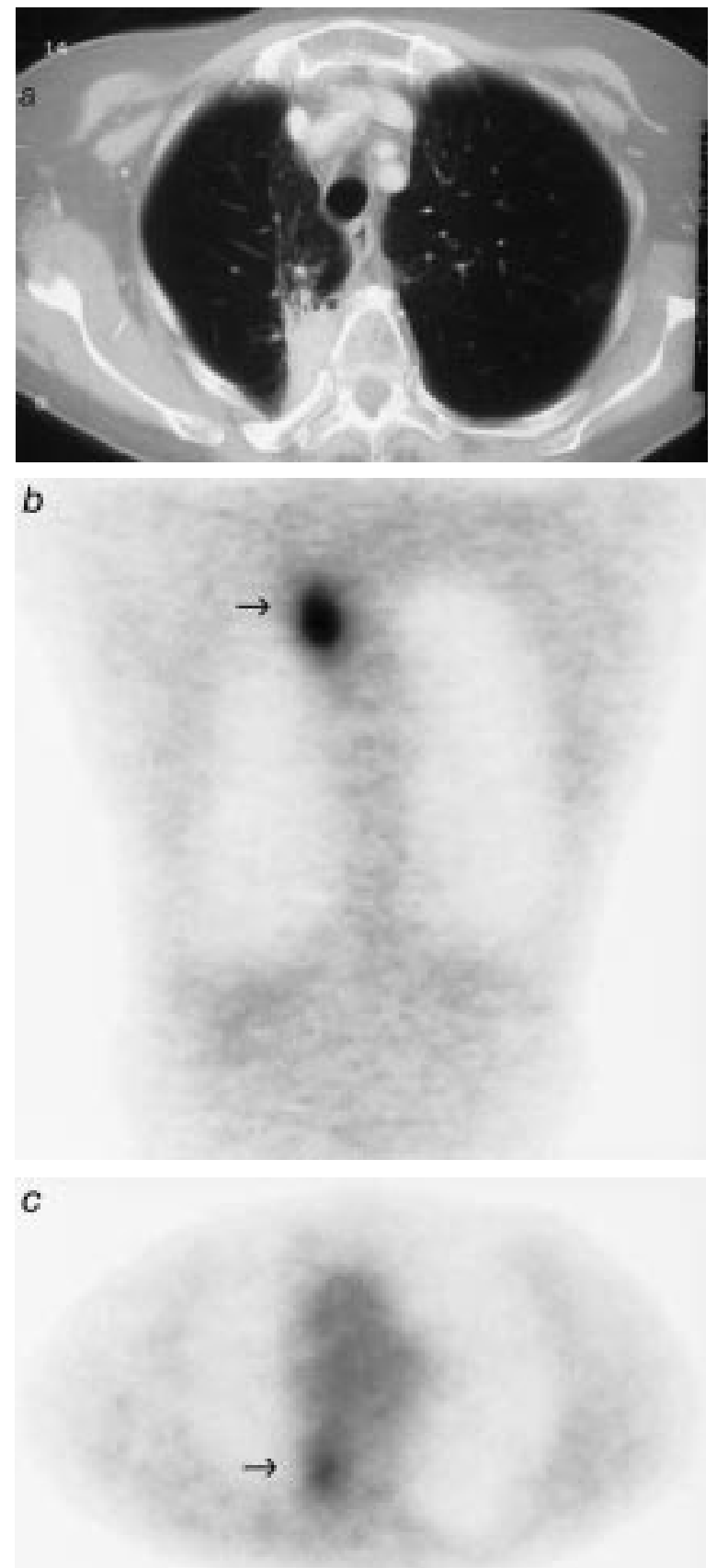

Fig. 1. - Discordance between computed tomography (CT) and positron emission tomography (PET) findings in a 74-yr-old female with a recurrent tumour observed 1 yr post-treatment. a) CT findings: stable CT image considered a radiation fibrosis and demonstration of parenchymal consolidation with a straight lateral margin, in the irradiated volume. b, c) PET findings: b) coronal, and c) transverse images showing 18-fluorodeoxyglucose uptake at the site (arrow) of the primary tumour (paramediastinal area in the right upper lobe). A transthoracic needle aspiration biopsy confirmed the recurrence of the tumour. radiographs or CT scans progressing gradually from pneumonitis to radiation fibrosis over a period of $\geq 6$ months were observed in 15 patients. Among these patients, only three had a positive PET scan (false positive cases), whereas 12 had a negative PET scan (true negative cases).

\section{Discussion}

FDG-PET is a noninvasive technique that appears to be very efficient in the staging of NSCLC. The results of the present study suggest that FDG-PET is also more accurate $(p=0.05)$ than CT in the detection of residual or recurrent lung cancer after treatment. From a clinical point of view, it may be difficult to differentiate recurrent tumours from post-treatment changes by means of morphological imaging since both processes may demonstrate similar appearances on a CT scan [9-11]. Therefore, some patients must undergo an invasive procedure in order to determine the persistence of viable tumour. A new noninvasive technique with the ability to clearly diagnose a recurrent tumour would thus be valuable.

In the present study, FDG-PET appeared to be more accurate than conventional imaging in distinguishing persistent or recurrent tumour from fibrotic scar tissue in patients who underwent treatment for NSCLC. FDG-PET correctly identified response to therapy in $96 \%$ (121 of 126) of patients. In particular, all recurrences $(n=60)$ were correctly identified by PET, whereas morphological imaging was noncontributory in $17(28 \%)$ cases at the time of PET diagnosis. Thus, in these cases, PET allowed the early detection of tumour recurrence before it became clinically apparent through other standard techniques. In the presentation of the results of this study, a distinction has been made between patients treated with early curative or palliative results because the authors believe that early detection of tumour recurrence could prove more useful for adjusting treatment in the hope of improving quality of life and survival in patients treated with curative intent. However, this point needs to be determined by a further study with a larger group of patients because the design of the present study and the timing of the use of the imaging modalities were probably not optimal for comparing early detection of tumour recurrence between PET and CT.

In the present study, 73 patients received treatment with radiotherapy and, of these, three gave a false positive PET result due to radiation pneumonitis during followup. In each case, patients received conventional radiotherapy with cobalt-60 to a total tumour dose of $60 \mathrm{~Gy}$ (4 Gy. fraction ${ }^{-1}, 12 \mathrm{~Gy} \cdot \mathrm{week}^{-1}$ ). When repair mechanisms take over following therapy, macrophages replace tumour cells and can also show FDG uptake. Indeed, FDG is not tumour-specific and determination of the optimal timing showing the effects of therapy without the pitfall of nonspecific uptake by macrophages justifies further research [12]. In

Table 4. - Comparative performances of computed tomography (CT) and positron emission tomography (PET) in the detection of residual of recurrent non-small cell lung cancer $(n=126)$

\begin{tabular}{lcccrr}
\hline & Sensitivity \% (n) & Specificity \% (n) & PPV \% (n) & NPV \% (n) & Accuracy \% (n) \\
\hline CT & $71.6(43 / 60)$ & $95.4(63 / 66)$ & $93.4(43 / 46)$ & $78.7(63 / 80)$ & $84.1(106 / 126)$ \\
PET & $100(60 / 60)$ & $92.4(61 / 66)$ & $92.3(60 / 65)$ & $100(61 / 61)$ & $96(121 / 126)$ \\
\hline
\end{tabular}

PPV: positive predictive value; NPV: negative predictive value. 
the authors' experience, the persistent too uptake associated with postirradiation pneumonitis is variable in intensity and also in kinetics and occasionally can last for 15 months after the end of therapy. This fact may complicate the early clinical follow-up of a few patients and justify a biopsy in order to determine tumour viability.

In this particular situation, sequential and semiquantitative PET scans could provide additional information in comparison with visual interpretation of PET data, to better distinguish inflammatory effects caused either by radiation or by tumour recurrence. However, preliminary reports of semiquantitative PET studies $[13,14]$ are conflicting. PATZ et al. [13] reported a 98\% diagnostic accuracy of FDGPET in the detection of recurrent lung tumours with a threshold SUV of 2.5, whereas INOUE et al. [14] also found a high sensitivity but found a relatively lower specificity with a threshold SUV of 3. In the three patients with radiation pneumonitis in the present study, semiquantitative analysis with a threshold SUV of 3 did not permit the distinction of pneumonitis from recurrent tumour. The authors would recommend that postradiotherapy PET is not performed earlier than 6 months after therapy in order to reduce the likelihood of false positive PET results.

The present study is in concordance with three earlier reports realised on a limited number of patients $(43,38$ and 20 (total 101), which suggested that FDG-PET is more precise than CT in the early detection of recurrent lung cancer [13-15]. In these reports, only one false negative PET result was described, in an asymptomatic patient who had biopsy-proven recurrent bronchoalveolar cell carcinoma 12 months after surgery [13]. In the present larger series, there were no false negative PET case; every patient with demonstrated lung tumour recurrence had a positive PET result at the time of diagnosis.

From experience, the authors believe that FDG-PET can play an important role in guiding patient care after treatment for lung cancer. The high NPV of PET suggests that residual abnormalities on $\mathrm{CT}$ and normal findings with PET scans are indicative of a true local remission of disease and do not justify a biopsy but, naturally, a follow-up. All of the patients in the present study achieving a complete response on the basis of PET results remained in remission for $>6$ months. Conversely, increased FDG uptake in suspicious lesions seen on CT is most probably caused by residual or recurrent lung cancer, even if a confirmatory biopsy may sometimes be required before additional therapy. The prognostic value of PET and its cost-effectiveness in the follow-up of lung cancer justify further research.

Therefore, FDG-PET imaging could be considered a very sensitive technique, the specificity of which is imperfect and should be compensated for by rigorous correlation with anatomical images (including image fusion, whenever possible). It is also possible that other tracers would be more appropriate than FDG in the monitoring of lung tumour treated by radiotherapy. In a preliminary report ( $\mathrm{n}=21)$, КUвотA et al. [16] suggested that $\mathrm{L}^{-11} \mathrm{C}$-methylmethionine (MET) uptake by the tumours showed a rapid response to radiotherapy, allowing better treatment follow-up with PET using MET. Further research is required to compare the usefulness of several tracers in differentiating radiation pneumonitis from tumour recurrence.
In conclusion, in the field of thoracic oncology, 18fluorodeoxyglucose positron emission tomography has been used to date essentially in the pretherapeutic staging of non-small cell lung cancer, but the authors believe that 18-fluorodeoxyglucose positron emission tomography is also useful in complementing computed tomography in the treatment follow-up of patients with non-small cell lung cancer because of its high sensitivity and negative predictive value.

\section{References}

1. Warburg O. On the origin of cancer cells. Science 1956; 123: 309-314.

2. Rege S, Hoh C, Glaspy J, et al. Imaging of pulmonary mass lesions with whole-body positron emission tomography and fluorodeoxyglucose. Cancer 1993; 72: 82-90.

3. Nolop K, Rhodes C, Brudin L, et al. Glucose utilization in vivo by human pulmonary neoplasms. Cancer 1987; 60: 2682-2689.

4. Bury T, Dowlati A, Paulus P, et al. Evaluation of the solitary pulmonary nodule by positron emission tomography imaging. Eur Respir J 1996; 9: 410-414.

5. Bury T, Paulus P, Dowlati A, et al. Staging of the mediastinum: value of PET imaging in non-small cell lung cancer. Eur Resp J 1996; 9: 2560-2564.

6. Bury T, Dowlati A, Paulus P, et al. Whole-body 18FDG positron emission tomography in the staging of lung cancer. Eur Resp J 1997; 10: 2529-2534.

7. Mountain CF. Revisions in the International System for Staging Lung Cancer. Chest 1997; 111: 1710-1717.

8. Cohen J. A coefficient of agreement for nominal scales. Educ Psychol Meas 1960; 20: 37-46.

9. Glazer H, Lee J, Levitt $\mathrm{R}$, et al. Radiation fibrosis: differentiation from recurrent tumor by MR imaging. Radiology 1985; 156: 721-726.

10. Glazer H, Aronberg D, Sagel S, Emami B. Utility of CT in detecting postpneumectomy carcinoma recurrence. $\mathrm{Am} \mathrm{J}$ Roentgenol 1984; 142: 487-494.

11. Bourgouin P, Cousineau G, Lemire P, Delvecchio P, Hebert G. Differentiation of radiation-induced fibrosis from recurrent pulmonary neoplasm by CT. J Can Assoc Radiol 1987; 38: 23-26.

12. Pauwels E, McCready R, Stoot J, Van Deurzen D. The mechanisms of accumulation of tumour-localising radiopharmaceuticals. Eur J Nucl Med 1998; 25: 277-305.

13. Patz E, Lowe V, Hoffman J, Paine S, Harris L, Goodman P. Persistent or recurrent bronchogenic carcinoma: detection with PET and 2-[F-18]-2-Deoxy-D-glucose. Radiology 1994; 191: 379-382.

14. Inoue T, Kim E, Komaki R, et al. Detecting recurrent or residual lung cancer with FDG-PET. J Nucl Med 1995; 36: 788-793.

15. Frank A, Lefkowitz D, Jaeger S, et al. Decision logic for retreatment of asymptomatic lung cancer recurrence based on positron emission tomography findings. Int $J$ Radiation Oncology Biol Phys 1995; 32: 1495-1512.

16. Kubota K, Yamada S, Ishiwaka K, et al. Evaluation of the treatment response of lung cancer with positron emission tomography and L-[methyl-11C] methionine: a preliminary study. Eur J Nucl Med 1993; 20: 495-501. 